\title{
Knowledge and practices of doctors at the National Hospital of Sri Lanka on screening and management of alcohol misuse among patients \\ KC Nagodavithana ${ }^{1}$, $\mathbf{N}$ Gunawardena ${ }^{2}$
}

\section{Abstract \\ Introduction}

Problems related to alcohol misuse in Sri Lanka are on the rise. Early screening and structured interventions by doctors have been shown to make a significant diminution in alcohol misuse among patients.

\section{Objectives}

This study aimed to assess the knowledge and practices of doctors at the National Hospital of Sri Lanka on screening and management of alcohol misuse among patients.

\section{Methods}

This was a hospital based descriptive cross-sectional study. Doctors $(n=385)$ of all designations attached to wards where patients with alcohol related problems are admitted, were assessed using a self-administered questionnaire. Factors associated with practices were assessed using cross tabulations and $\chi^{2}$ statistic.

\section{Results}

Response rate was high $(91.4 \%)$. Respondents were mainly males $(63.6 \% ; \mathrm{n}=224)$ in the age group of $25-34$ years $(48.0 \%$; $n=169)$. A majority were in the category of 'medical officers' $(39.4 \% ; \mathrm{n}=139)$ attached to general medicine or surgery units $(49.7 \% ; \mathrm{n}=175)$ with work experience of $<10$ years $(68.2 \%$; $n=240)$.

A weighted score was developed for overall knowledge on screening and management. The proportion of doctors with 'Good' overall knowledge was high $(75.9 \% ; n=267)$. However, only $53.4 \%(\mathrm{n}=188)$ were aware of available standard screening tools.

The majority $(93.5 \%$; $n=329)$ of doctors were inquiring into alcohol habits of male patients during their routine practice. However, those utilizing standard screening tools to determine alcohol misuse was low $(22.8 \% ; \mathrm{n}=75)$.

Though the proportion of doctors who took steps to reduce misuse was high $(80.3 \%$; $n=283)$, the steps that would achieve sustainable reduction were taken by only $45.9 \%(\mathrm{n}=130)$.

'Good' overall knowledge $(\mathrm{p}<0.001)$, working in a medical unit $(\mathrm{p}<0.001)$, work experience $<10$ years $(\mathrm{p}<0.001)$, were associated with 'always inquiring' into alcohol habits in routine practice while 'Good' overall knowledge ( $<<0.001)$, being a house officer $(\mathrm{p}<0.001)$ and work experience $<10$ years $(\mathrm{p}<0.001)$ were associated with taking steps to reduce misuse.

\section{Conclusion and Recommendations}

Overall knowledge was found to be 'Good'. Gaps in practice of screening and management were evident, which need to be corrected.

\section{Key Words}

Alcohol misuse, screening, management methods

1. MD Trainee in Community Medicine, Post Graduate Institute of Medicine, University of Colombo

2. Senior Lecturer, Department of Community Medicine, Faculty of Medicine, University of Colombo 


\section{Introduction}

Alcohol misuse can cause physical and psychological problems to the individuals (1). The problems related to alcohol misuse can have an impact on the individual who consumes alcohol, on family members

(especially the spouse and the children), and there can be consequences to the society as a whole (2).

A large number of people who misuse alcohol are admitted to hospitals with either a considerable number of doctors of various specialities of medicine including general medicine, general surgery, accident service, orthopaedic, cardiology, cardiothoracic surgery, neurology, psychology, neurosurgery and gastroenterology attend to their presenting problem and provide treatment as needed.

There is reasonable doubt whether the doctors who treat them, inquire into the alcohol consumption patterns of these patients and identify alcohol misuse. In most instances, patients with alcohol dependence are identified and managed only when they present with related medical problems. Then the patients are referred to a psychiatry unit for further management (4).

It has been found that early screening of alcohol misuse among patients and adoption of evidence based management steps will lead to sustainable reduction in alcohol misuse among the patients (5).

Information regarding the practices of the doctors in the ward setup on screening and managing patients with alcohol misuse is very scarce in Sri Lanka.

This study aimed at finding out the extent to which the doctors in the hospital setup are involved in screening and management of alcohol misuse of patients, their knowledge, attitude and practices for doing so. Association between selected factors and practices of screening and management was also assessed.

\section{Methodology}

This descriptive cross sectional study was conducted at the National Hospital of Sri Lanka (NHSL). The NHSL was selected as the study setting for the present study, because a considerable number of patients with various problems due to misuse of physical, psychological problems or accidental and non-accidental injuries (3) alcohol are admitted (6). Being a teaching hospital, all categories of doctors including post graduate trainee doctors were available to be included in the study.

The study population was considered as all the doctors (Consultants, senior registrars, registrars, medical officers, and house officers) who are directly involved in the management of patients and have the opportunity to communicate with patients who are admitted to wards with problems related to alcohol misuse. All the doctors attached to the wards of general medicine, general surgery, accident service, orthopaedic, cardiology, cardiothoracic surgery, psychiatry, neurology, gastroenterology and burns unit were considered as eligible to be included in the study.

It was decided to include all the doctors $(n=385)$ who were attached to those relevant unit of NHSL. Data collection was done during the study period extending from August to November of 2008.

The numbers of eligible doctors and the ward/unit to which they are attached to and their designation was obtained from the updated data base of administration unit of NHSL. Each of these wards/units was visited and a brief introduction of the study along with an information sheet was provided to each of the selected study participant personally by the principal investigator and they were invited to participate in the study. 
A pre tested, self administered questionnaire was used to obtain data related to socio demographic and work related information, Knowledge on detection and management of alcohol misuse, attitudes and self reported information pertaining to practices on screening and management.

The data were analysed using Statistical Package for Social Sciences version 16. Ethical clearance was obtained from the Ethical Review Committee of the Faculty of
Medicine, University of Colombo and administrative approval was obtained from the Director of NHSL. Informed verbal consent was obtained from each participant. Ensuring anonymity, the confidentiality of the information that was supplied was stressed.

\section{Results}

Study included 352 doctors with a response rate of $91.4 \%(352 / 385)$. No significant differences were seen between the respondents and the non respondents after analysing preliminary data.

Table 1 describes the socio demographic characteristics of the study participants.

Table 1-Distribution of demographic characteristics of the study population

\begin{tabular}{lcc}
\hline Characteristic & $\begin{array}{c}\text { Frequency } \\
(\mathbf{n = 3 5 2})\end{array}$ & Percentage \\
\hline Sex & 224 & 63.6 \\
Male & 128 & 36.4 \\
Female & & \\
Age (Years) & 169 & 48.0 \\
25 to 34 & 137 & 38.9 \\
35 to 44 & 41 & 11.6 \\
45 to 54 & 5 & 1.4 \\
$\geq 55$ & & \\
Ethnicity & 283 & 80.4 \\
Sinhala & 53 & 15.1 \\
Tamil & 16 & 4.5 \\
Muslim & 0 & 0 \\
Burger & & \\
\hline
\end{tabular}


A majority were in the category of 'medical officers' $(39.4 \% ; n=139)$ attached to general medicine or surgery units $(49.7 \% ; \mathrm{n}=175)$ with work experience of $<10$ years $(68.2 \%$; $\mathrm{n}=240)$. Many (31.8\%) have seen a total of 31-50 new patients on average per week while nearly one fourth (23.9\%) have encountered 51-70 new patients per week.

A weighted score was developed for the assessment of the overall knowledge on detection and management of alcohol misuse, of which 0-60 was taken as 'poor' and 61-100 as 'good'. Approximately fourth $(75.9 \%)$ had a 'good' level of overall knowledge. But only 188 (53.5\%) were aware of a standard screening method (e.g. screening tool) that can be used to detect alcohol misuse. The screening method that was known by a majority (72.3\%) was the CAGE questionnaire while $27.7 \%$ were aware of the AUDIT questionnaire.

The majority $(84.0 \%)$ of that group have stated that they were made aware of screening methods to detect alcohol misuse during their undergraduate training.

Distribution of the awareness on the available management options that can be used to reduce alcohol misuse is described in Table 2.

Table2-Distribution of the awareness of the study population on the available management options that can be used to reduce alcohol misuse

\begin{tabular}{lcc}
\hline Management option & $\begin{array}{c}\text { Frequency } \\
(\mathbf{n = 3 5 2})\end{array}$ & Percentage \\
& 215 & 61.1 \\
\hline Drug treatment & 293 & 83.2 \\
Simple advice to cut down drinking & 311 & 88.4 \\
Educating on the ill effects to health & 295 & 83.8 \\
Counsel to reduce risk drinking & 297 & 84.4 \\
Refer for specialized care & 269 & 76.4 \\
Involving family members in the management & &
\end{tabular}

More than one response was allowed Approximately half $(49.1 \%)$ of the study population reported that they always make inquiries into the alcohol habits of male patients during their routine ward work while $37.5 \%$ stated that they make these inquiries only from the patients for whom they consider it necessary based on the circumstances of admission. 
Looking into their practices, a total of 329 $(93.5 \%)$ participants in the study population circumstances or if the patient himself indicates of an alcohol problem.

Furthermore, among the 75 doctors who have utilized a patient and a situation.

Among those doctors, only $22.8 \% \quad(n=75)$ use a standard screening questionnaire while more than half $(61.7 \%)$ ask according to the standard screening questionnaire, the CAGE questionnaire was used by the majority $(82.67 \%)$ but that percentage out of the total respondents is low $(21.3 \%)$.

Most of the respondents reported that they have assessed the amount consumed $(86.4 \%)$ and the duration of use of alcohol by patients $(85.8 \%)$. Status of dependence was assessed by $73.9 \%$. Physical, social and psychological problems were assessed by $56.8 \%, 52.8 \%$ and $46.9 \%$ respectively. Only $35.5 \%$ inquired regarding the amount spent for alcohol misuse and attempts to quit in the past. Nearly half $(46.8 \%)$ of the study participants who made inquiries into alcohol habits utilized 3-5 minutes to screen patients while only $1.8 \%$ used more than 10 minutes to do so.

The study population were asked whether in their routine practice they take any steps to reduce the misuse among patients with alcohol misuse, a total of $283(80.3 \%)$ responded positively. Among the respondents a majority $(93.6 \%)$ educated the patients on the ill effects on health. Counselling to change behaviour and referrals to specialized care was carried out by $45.8 \%$ and $42.8 \%$ respectively as reported by the respondents.

Table 3 describes the barriers identified by the study population to carrying out screening and management to reduce alcohol misuse in the ward setup.

Table 3-Distribution of the study population who identified barriers to screen and manage alcohol misuse among patients in the ward setup by their responses

\begin{tabular}{lcc}
\hline Identified barriers & Frequency $(\mathbf{n = 2 8 0})$ & Percent \\
\hline Lack of time & 248 & 88.6 \\
Inadequate training in detecting & 91 & 32.5 \\
Language barriers & 55 & 19.6 \\
Lack of interest & 45 & 16.1 \\
Lack of privacy in the ward & 108 & 38.6 \\
Inadequate training to manage & 112 & 40.0 \\
No authority to prescribe & 40 & 14.3 \\
Lack of communication skills & 37 & 13.2 \\
\hline
\end{tabular}


More than one response was allowed Association between making inquiries into alcohol habits in routine practice when encountered with a male patient and selected factors as well as the association between taking steps to reduce alcohol misuse and selected factors were assessed by cross tabulation and by applying chi-squared test. The findings are summarized in Tables 4 and 5 respectively.

Table 4 -Association between making inquiries into alcohol habits in routine practice and selected factors

\begin{tabular}{lccc}
\hline Selected factor & $\chi^{2}$ & df & p value \\
\hline Having a ‘Good' Overall knowledge & 15.44 & 1 & $\mathrm{p}<0.001$ \\
Being attached to a general medical unit & 16.08 & 2 & $\mathrm{P}<0.001$ \\
Work Experience of less than 10 years & 13.49 & 1 & $\mathrm{P}<0.001$ \\
Male doctors who consume alcohol at present & 1.21 & 1 & $\mathrm{p}=0.27$ \\
\hline
\end{tabular}

Table 5 -Association between taking steps to reduce alcohol misuse and selected factors

\begin{tabular}{lccc}
\hline Selected factor & $\chi^{2}$ & Df & p value \\
\hline Having a 'Good' Overall knowledge & 58.29 & 1 & $\mathrm{p}<0.001$ \\
Being a house officer & 22.55 & 3 & $\mathrm{P}<0.001$ \\
Work Experience of less than 10 years & 16.39 & 1 & $\mathrm{P}<0.001$ \\
Male doctors who consume alcohol at present & 1.64 & 1 & $\mathrm{p}=0.2$ \\
\hline
\end{tabular}

\section{Discussion}

The number of wards of general medicine and general surgery is higher than the other specialities in the study setting, so is the number of doctors attached to those units, which justifies the representation of approximately half $(49.7 \%)$
The study population from those two specialities combined. These are the units to which most of the patients with direct or indirect effects of misuse of alcohol get admitted for care. 
The fact that more than half $(57.7 \%)$ of the study population who were attached to general medicine, general surgery and accident service come across a total of 31 or more new patients per week, reflects the increased opportunity presented for a majority of doctors attached to those units to screen for alcohol misuse.

On the other hand, one can also argue that when the average number of patients increases the average time that can be devoted for each patient may be reduced which may affect the practice of screening and management related to alcohol misuse, which is evident by the fact that nearly half $(46.8 \%)$ of the study participants who made inquiries into alcohol habits utilized 3-5 minutes to screen patients while only $1.8 \%$ used more than 10 minutes to do so.

In the present study, $53.4 \%$ of the population were aware of a standard screening questionnaire for detecting alcohol misuse among patients which is better compared to the study conducted among GPs' in 2 districts in Sri Lanka revealed that the awareness on screening tools were low (25.7\%) (7). Even though study groups are not directly comparable, this may be due to the increased opportunities of learning available in a teaching hospital in the present study.

Among the respondents in the present study who knew of a standard screening tool, the majority $(72.3 \%)$ were aware of the CAGE questionnaire which is a reliable and valid screening tool (8) that can be applied quickly and easily in a ward setup (9). However, awareness on the evidence of the effectiveness of this tool among them was low $(51.1 \%)$. The most $(84 \%)$ cited source of information on the screening methods was undergraduate training even though approximately one third of the doctors had practiced for more than 10 years after their undergraduate training.

The proportion of the study population with 'Good' overall knowledge on screening and management of alcohol misuse was high $(75.9 \%)$. This indicates that majority have the correct knowledge regarding the conditions which should trigger a doctor to inquire into alcohol use in a patient and to take steps to reduce.

It was encouraging to observe that a majority $(93.5 \%)$ of the study population was making inquiries in to alcohol habits of male patients during their routine practice. However, only half $(49.1 \%)$ practice that 'Always', highlighting the potential for improvement of the current practices in order to encourage doctors to always inquire into alcohol habits as recommended by the National Institute of Alcohol Abuse and Alcoholism (10). The cross sectional study conducted among GPs' in Colombo and Gampaha districts revealed that only $15 \%$ of the respondents had routinely inquired about alcohol use (7) while in another study among a random sample of surgeons in the United States, routine screening was found to be low (11). These are in contrast to the present study.

Among those who inquired into alcohol habits, the proportion of the study participants utilizing standard screening questionnaires to determine alcohol misuse was low (22.8\%) despite the fact that more than half the respondents were reported as being aware of the screening tools. Regarding the steps that doctors take to reduce alcohol misuse, a majority $(93.6 \%)$ of the study population educated the patients on the ill effects of alcohol on health. 
Simple education as such has been proven to reduce the alcohol consumption among patients (12) but this has not shown long term sustainability of reduction of misuse. Brief counselling is a method that has been proven to achieve long term sustainable reduction of misuse of alcohol (13) which was reported as being practised by a low proportion $(45.9 \%)$ of the doctors in the present study. This reflects need to develop the doctors' counselling practices.

Regarding the barriers identified for screening and management of alcohol misuse in the ward setup, a higher proportion $(88.6 \%)$ identified inadequacy of time as the barrier. Therefore, this strengthens the fact that training doctors on quick, easy methods of screening. Inadequacy in training to detect and inadequacy in training to manage alcohol misuse was considered as barriers by $32.5 \%$ and $40 \%$ of the study participants respectively. This further justifies the need for training programmes for the doctors on screening and management on alcohol misuse.

\section{Conclusions and recommendations}

Doctors possess a good overall knowledge on screening and management of alcohol misuse; but with a low awareness of screening tools such as CAGE and AUDIT and their effectiveness. Awareness should be enhanced on the currently available screening tools to detect alcohol misuse and brief interventions that can be utilised in a ward set up.

Knowledge on screening and management were mostly achieved during undergraduate training and a scarcity of continuous medical education opportunities on the subject was noted indicating that there should be programmes of continuous medical education for revision and gathering of knowledge.

The proportion of doctors who always inquired into alcohol misuse when encountered with a patient and the use of screening tools for this purpose was low. The recommendation of the National Institute of Alcohol and Alcoholism on always inquire into alcohol habits should be conveyed to the doctors.

The proportion of doctors who adopt management methods that are proved to have long term effects to reduce consumption was low. Doctors should be offered more opportunities of developing their counselling skills to improve long term reduction of alcohol misuse among patients.

\section{Acknowledgement}

Education, Training and Research unit of Ministry of Health for provision of financial assistance.

\section{References}

1. World Health Organization. Prevention of harm from alcohol use, get high on life without alcohol. 2003. New Delhi: Regional Office for South-East Asia, World Health Organization.

2. World Health Organization. The World Health Report 2002 - Reducing Risks, Promoting Healthy Life Geneva: World Health Organization.

3. Anderson P, Cremona A, Paton A, Turner C, Wallace P. The risk of alcohol. Addiction 1993; 88: 1493-1508. 
4. World Health Organization. Fifty Eight World Health Assembly - Fourth report of committee B 2005. Geneva, World Health Organization: A 58/61. http://www.who.int/gb/ebwha/pdf_files/WH A58/A58_61-en.pdf [Accessed 17 July 2008].

5. Babor TF and Higgins-Biddle JC. Brief intervention for hazardous and harmful drinking: a manual for use in primary care. 2001. Geneva: World Health Organization.Ministry of Health. Annual Health Bulletin 2003. Colombo: Ministry of Health.

6. Gurugama NP, Seneviratne SL, Peiris DTS, Silva HJ. Detection and management of alcohol misuse by general practitioners. Ceylon Medical Journal 2003; 48(4): 122-124.

7. Fiellin DA, Reid MC, O'Connor PG. Screening for alcohol problems in primary care: Systemic review. Journal of General Internal Medicine 2000; 15(1): 65-66.

8. Ewing JA. Detecting alcoholism: The CAGE questionnaire. Journal of the American Medical Association 1984; 252: 1905-1907.

9. National Institute on Alcohol Abuse and Alcoholism. The physicians' guide to helping patients with alcohol problems. 1995. Washington, D.C.: Government Printing Office, (NIH publication no. 95-3769.)
10. Danielsson E, Fredrick PR, Larry MG, Ronald VM. Reasons why trauma surgeons fail to screen for alcohol problems. Archives of Surgery 1999; 134: 564-568.

11. Wallace P, Cutler S, Haines A. Randomized controlled trial of general practitioner intervention in patients with excessive alcohol consumption British Medical Journal 1988;297: 663-668.

12. Jonathan C, Geoffrey L, Evelyn C. Counselling problem drinkers in medical wards: a controlled study. British Medical Journal 1985; 290: 965-967. 